\title{
Successful primary percutaneous coronary intervention in patient with ST-segment elevation myocardial infarction via left snuffbox approach: Patient advantages
}

\author{
Yongcheol Kim, Myung Ho Jeong, Min Chul Kim, Doo Sun Sim, \\ Young Joon Hong, Ju Han Kim, Youngkeun Ahn \\ Department of Cardiology, Chonnam National University Hospital, Gwangju, Republic of Korea
}

A 40-year-old man was referred to the documented institute with evidence of ST-segment elevation in the anterior electrocardiogram leads. Urgent coronary angiography (CAG) was performed via the left snuffbox approach using $6 \mathrm{Fr}$ sheath (Fig. 1A) with loading of acetylsalicylic acid $300 \mathrm{mg}$ and prasugrel $60 \mathrm{mg}$. After administration of 3000 units unfractionated heparin via sheath, CAG demonstrated total occlusion in the proximal left anterior descending artery (LAD) (Fig. 1B). Primary percutaneous coronary intervention $(\mathrm{PCI})$ was successfully performed with a $3.25 \times 23 \mathrm{~mm}$ everolimus-eluting stent after intracoronary bolus of abciximab (Fig. 1C). Hemostasis was obtained by compressive bandage method after PCI and he was moved to the coronary care unit (CCU). There was no puncture site complication after hemostasis for $3 \mathrm{~h}$ (Fig. 1D) and the patient did not complain about an inconvenience of finger or wrist movement in the puncture side hand after removal of the sheath (Fig 1E). Post-PCI echocardiography demonstrated hypokinesia of the anterior wall with good left ventricular systolic function. Two days subsequent to PCI, including a 1-day CCU and 1-day ward care, the patient was discharged without any sequelae.

As shown in Figure 2, there are 2 snuffbox puncture sites where well pulsation of the distal radial artery can be found. One is located in the anatomical snuffbox and the other is in the first intermetacarpal space, which is the proximal area from Trapezoid and Trapezium [1]. Data regarding the snuffbox approach remains limited, but published data and cases supporting that snuffbox approach could be considered as an alternative access site in selected patients, such as patients with high bleeding risk or renal impairment $[2,3]$. Especially, the left snuffbox approach might be useful for elderly patients due to less subclavian tortuosity when compared with the right radial approach [4]. In terms of feasibility of the snuffbox approach for primary PCI, prospective studies are needed to confirm safety and efficacy as there has been only 1 case report published [5]. Furthermore, there are no data regarding the benefit of the snuffbox approach for early discharge, even though current guidelines support that early discharge, within 48-72 h, should be considered in selected low-risk patients with ST-segment elevation myocardial infarction (STEMI) after successful primary PCI. This case illustrates the potential benefits of the snuffbox approach regarding hemostasis and early mobilization leading to the early discharge after successful primary PCI in the low-risk patient with STEMI.

Conflict of interest: None declared

\footnotetext{
Address for correspondence: Myung Ho Jeong, MD, Cardiovascular Convergence Research Center of Chonnam National University Hospital, Gwangju 61469, Republic of Korea, tel: 82-62-220-6243, fax: 82-62-228-7174, e-mail: myungho@chollian.net
} 


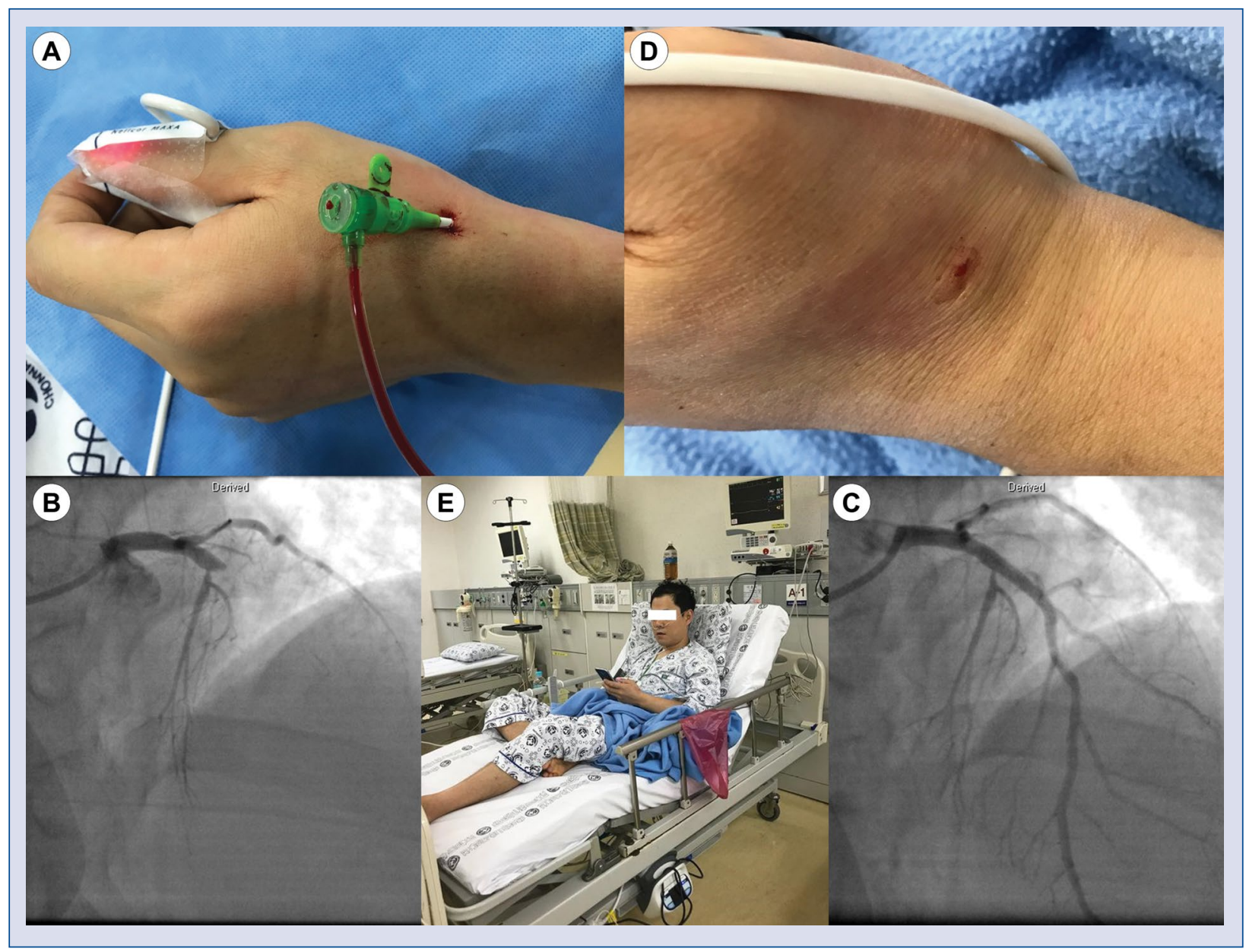

Figure 1. A. Inserted $6 \mathrm{Fr}$ sheath via left snuffbox approach; B. Urgent coronary angiography (CAG) demonstrating thrombotic total occlusion in the proximal left anterior descending artery; C. CAG demonstrating successful primary percutaneous coronary intervention with drug-eluting stent implantation; D. No bleeding complication of puncture site after 3-hour hemostasis; E. Patient using a mobile phone without the inconvenience of puncture-side hand immediately after removal of sheath.

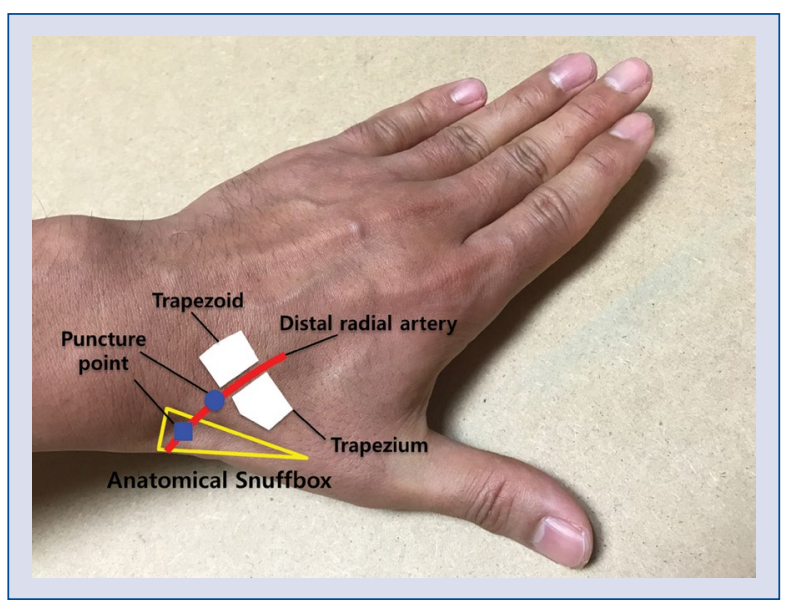

Figure 2. Snuffbox puncture sites in the anatomical snuffbox (blue box) and in the first intermetacarpal space (blue circle).

\section{References}

1. Roh JH, Lee JH. Distal radial approach through the anatomical snuff box for coronary angiography and percutaneous coronary intervention. Korean Circ J. 2018; 48(12): 1131-1134, doi: 10.4070/kcj.2018.0293, indexed in Pubmed: 30403016.

2. Kim Y, Ahn Y, Kim I, et al. Feasibility of coronary angiography and percutaneous coronary intervention via left snuffbox approach. Korean Circ J. 2018; 48(12): 1120-1130, doi: 10.4070/ /kcj.2018.0181, indexed in Pubmed: 30088362.

3. Kim Y, Ahn Y, Kim MC, et al. Gender differences in the distal radial artery diameter for the snuffbox approach. Cardiol J. 2018; 25(5): 639-641, doi: 10.5603/CJ.2018.0128, indexed in Pubmed: 30394514.

4. Berezhnoi K, Kokov L, Vanyukov A, et al. Complete revascularization via left snuffbox approach in a nonagenarian patient with acute myocardial infarction. Cardiol J. 2018; 25(4): 530-531, doi: 10.5603/CJ.2018.0083, indexed in Pubmed: 30211930.

5. Kim Y, Jeong MH, Berezhnoi K, et al. Recannulation of Distal Radial Artery for Staged Procedure After Successful Primary Percutaneous Coronary Intervention. J Invasive Cardiol. 2018; 30(10): E105-E106, indexed in Pubmed: 30279299. 